\title{
A layout algorithm for undirected compound graphs is
}

\author{
Ugur Dogrusoz $^{\mathrm{a}, \mathrm{b}, *}$, Erhan Giral ${ }^{\mathrm{b}}$, Ahmet Cetintas ${ }^{\mathrm{a}}$, Ali Civril ${ }^{\mathrm{c}}$, Emek Demir $^{\mathrm{d}}$ \\ ${ }^{a}$ Computer Engineering Department, Bilkent University, Cankaya, 06800 Ankara, Turkey \\ ${ }^{\mathrm{b}}$ Tom Sawyer Software, Oakland, CA, USA \\ ${ }^{\mathrm{c}}$ Rensselaer Polytechnic Institute, Troy, NY, USA \\ ${ }^{\mathrm{d}}$ Memorial Sloan-Kettering Cancer Center, New York, NY, USA
}

\section{A R T I C L E I N F O}

\section{Article history:}

Received 7 May 2008

Received in revised form 5 November 2008

Accepted 10 November 2008

\section{Keywords:}

Information visualization

Graph drawing

Force-directed graph layout

Compound graphs

Bioinformatics

\begin{abstract}
A B S T R A C T
We present an algorithm for the layout of undirected compound graphs, relaxing restrictions of previously known algorithms in regards to topology and geometry. The algorithm is based on the traditional force-directed layout scheme with extensions to handle multilevel nesting, edges between nodes of arbitrary nesting levels, varying node sizes, and other possible application-specific constraints. Experimental results show that the execution time and quality of the produced drawings with respect to commonly accepted layout criteria are quite satisfactory. The algorithm has also been successfully implemented as part of a pathway integration and analysis toolkit named PATIKA, for drawing complicated biological pathways with compartmental constraints and arbitrary nesting relations to represent molecular complexes and various types of pathway abstractions.
\end{abstract}

(c) 2008 Elsevier Inc. All rights reserved.

\section{Introduction}

As graphical user interfaces have improved, and more state-of-the-art software tools have incorporated visual functions, interactive graph editing and diagramming facilities have become important components in visualization systems [7]. Effective analysis of the underlying data in graph visualization is only possible with the sound automatic layout capabilities of such systems.

The notion of compound graphs has been used, in the past, to represent more complex types of relationships or varying levels of abstractions in data (see Figs. 1 and 2) $[20,17,16,14]$.

There has been a great deal of work done on general graph layout [4] but considerably less on the layout of compound graphs, probably due to the difficult nature of the problem. Straightforward approaches to laying out compound graphs in a top-down or bottom-up manner (with respect to the inclusion or nesting hierarchy) fail, due to bidirectional dependencies (e.g., inter-graph edges) between levels of varying depth. The limited work on compound graph layout has mostly focused on the layout of hierarchical graphs $[19,18,9]$, in which the underlying relational information is assumed to be under a certain hierarchy. However, such algorithms perform poorly if the graph is undirected (if the edge directions do not enforce a hierarchy) but still has structural properties, like symmetry, or includes parts or substructures,

\footnotetext{
A short demo version of this paper appeared in Proc. of 12th Intl. Symposium on Graph Drawing, NYC, New York, pp. 442-447, September 29-October 2, 2004. Research supported in part by TUBITAK (The Scientific and Technological Research Council of Turkey), grant number $104 \mathrm{E} 049$.

* Corresponding author. Address: Computer Engineering Department, Bilkent University, Cankaya, 06800 Ankara, Turkey. Tel.: +90 (312) 290 1612; fax: +90 (312) 2664047

E-mail address: ugur@cs.bilkent.edu.tr (U. Dogrusoz).
} 


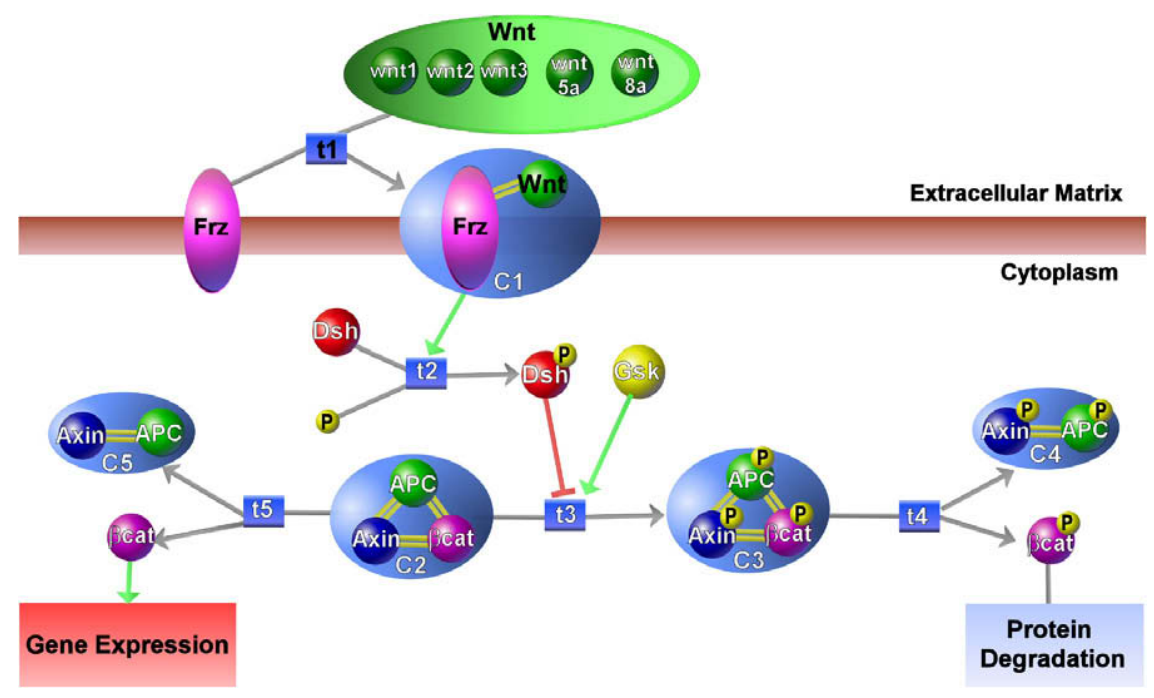

Fig. 1. Part of a sample compound pathway.

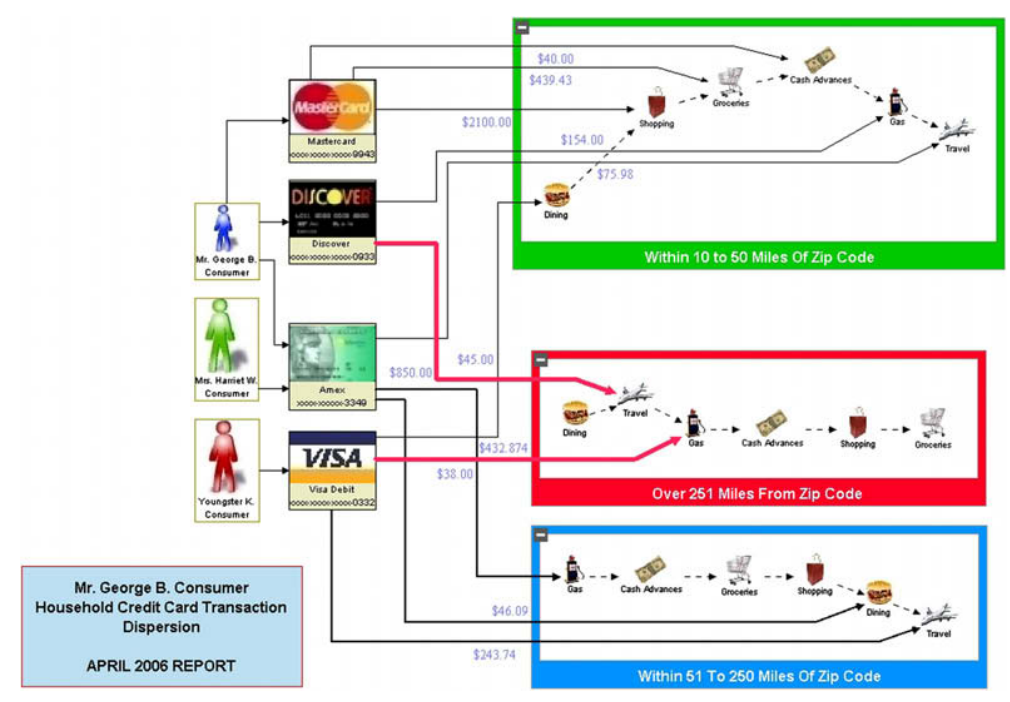

Fig. 2. A financial chart (courtesy of Tom Sawyer Software).

such as cycles. The work on undirected compound graphs $[1,21,12,10,3,5]$, on the other hand, is either restricted in the types of graphs addressed (e.g., clustered graphs, where grouping or nesting is allowed for only one level) or is unsatisfactory in terms of the quality of results produced (e.g., large compound nodes overlapping with others or an inefficient use of area).

In this paper, we describe a new algorithm for the layout of undirected compound graphs that overcomes the shortcomings of previous algorithms. Ours is based on the force-directed layout algorithm $[8,13]$ and is the first for drawing undirected compound graphs, to handle all of the following (Fig. 3) with a rather simple, intuitive, force-directed model:

- an arbitrary level of nesting,

- inter-graph edges that may span multiple levels of nesting, and

- links to non-leaf nodes in the nesting hierarchy. 


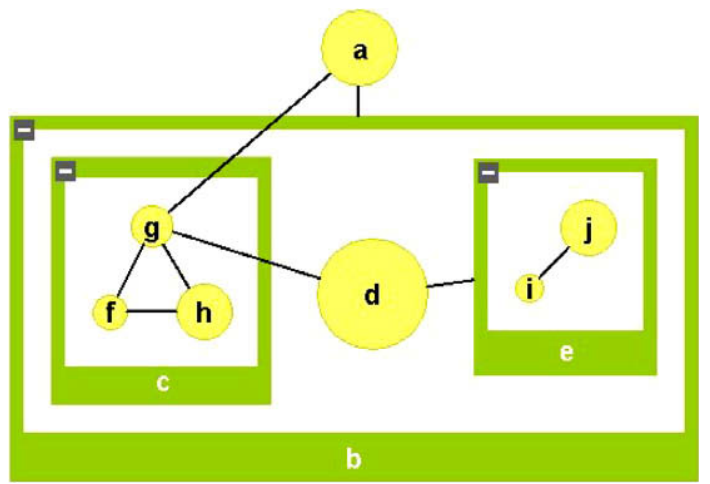

Fig. 3. An example of a compound graph with multiple levels of nesting (3), inter-graph edges spanning multi-levels (e.g., edge $\{a, g\}$ ), edges with non-leaf end-nodes (e.g., edge $\{d, e\}$ with non-leaf end node $e$ ), and varying node dimensions.

Furthermore, it can handle non-uniform node sizes.

The rest of this paper is organized as follows: the next section gives some definitions used throughout the paper. Then, we present our layout algorithm, detailing the idea behind the methodology, followed by its pseudo-code. We also discuss how application-specific constraints can be integrated into our algorithm. In addition, an implementation used to verify the quality and performance of the algorithm is discussed. This algorithm has also been implemented within the software tool PATIKA [6] to visualize complicated biological pathways with compartmental constraints and nested drawings.We conclude with a brief summary.

\section{Definitions}

We assume the reader is familiar with basic notation and definitions of graph theory. A node $v \in V$ (an edge $e \in E$ ), where $G=(V, E)$, is said to be a member of graph $G$; conversely, $G$ is said to be the owner of node $v$ (edge $e$ ). A compound graph $C=(V, E, F)$ consists of nodes $V$, adjacency edges $E$, and inclusion edges $F$. It is required that the inclusion graph $T=(V, F)$ is a rooted tree, and no adjacency edge connects a node to one of its descendants or ancestors. For instance, for the compound graph in Fig. 3,

$$
\begin{aligned}
& V=\{a, b, \ldots, j\} \\
& E=\{\{a, b\},\{a, g\},\{d, e\},\{d, g\},\{f, g\},\{f, h\},\{g, h\},\{i, j\}\}, \quad \text { and } \\
& F=\{b c, b d, b e, c f, c g, c h, e i, e j\} .
\end{aligned}
$$

For convenience, our implementation represents unrestricted undirected compound graphs with geometry information using a graph manager. A graph manager $M=(S, I, F)$ defined by a graph set $S=\left\{G_{1}, G_{2}, \ldots, G_{l}\right\}$, an inter-graph edge set $I$, and a rooted nesting tree $F=\left(V^{F}, E^{F}\right)$. With this representation, the topology of a compound graph is split into multiple graphs that are nested within each other. The geometry of each node is represented by a rectangle. The nesting of a graph in a node facilitates the drawing of multiple graphs of a graph manager and their interrelations simultaneously. The node within which a graph is nested, is said to be expanded. The size of an expanded node is as big as the boundaries of the associated nested graph. This is represented in the nesting tree by an edge $\left\{u, G_{i}\right\} \in E^{F}$ between a node $u$ and a graph $G_{i}$, where $G_{i}$ is not a (direct or indirect) owner of $u$. $G_{i}$ is said to be the child graph of the parent node $u$. The graph at the root of the nesting tree is simply called root graph.

Another way of associating two different graphs in a graph manager $M=(S, I, F)$ is via the inter-graph edge set $I$. Let $u \in V^{G_{i}}$ and $v \in V^{G_{j}}$ be two nodes, where $i \neq j$ and $G_{i}, G_{j} \in S$. Then, the edge $\{u, v\} \in I$ is called an inter-graph edge, representing a relation between nodes that belong to different entities, graphs $G_{i}$ and $G_{j}$ in this case.

For instance, for the compound graph in Fig. 3,

$$
\begin{aligned}
& G_{1}=(\{a, b\},\{\{a, b\}\}), \quad G_{2}=(\{c, d, e\},\{\{d, e\}\}), \\
& G_{3}=(\{f, g, h\},\{\{f, g\},\{f, h\},\{g, h\}\}), \quad G_{4}=(\{i, j\},\{\{i, j\}\}) \\
& S=\left\{G_{1}, G_{2}, G_{3}, G_{4}\right\}, \quad I=\{\{a, g\},\{d, g\}\}, \quad \text { and } \\
& F=\left(\left\{G_{2}, G_{3}, G_{4}\right\},\left\{b G_{2}, G_{2} c, G_{2} e, c G_{3}, e G_{4}\right\}\right) .
\end{aligned}
$$

The owner graph of nodes $f, g$, and $h$ is $G_{3}$, which in turn is the child graph of its parent node $c$. 


\section{Layout algorithm}

\subsection{Underlying physical model}

A basic force-directed layout algorithm with certain extensions to satisfy the general drawing conventions in compound graphs was chosen. The basic idea of the layout algorithm is to simulate a physical system in which nodes are assumed to be physical objects with a certain "electrical charge", connected via "springs" of a pre-specified desired length. Objects pull or repel each other depending on the current lengths of any connected springs. In addition, relatively minor repulsion forces act on any pair of objects that are "too close" to each other to avoid node-to-node overlaps. Furthermore, we assume "gravitational forces" to keep graph components together. In order to handle varying node sizes (especially expanded nodes) and to avoid overlaps with neighboring nodes, calculation of edge lengths are based on the parts of edges in between the borders of end-nodes, as opposed to their centers [15]. Thus, the optimal layout is regarded as the state of this system, in which the total energy is minimal. The following additions are made to this basic model:

- An expanded node and its associated nested graph are represented as a single entity, similar to a "cart", which can move freely in orthogonal directions (no rotations allowed). Multiple levels of nesting is modeled with smaller carts on top of larger ones (Fig. 4).

- The nodes and edges of a nested graph are to be set in motion on this cart, confined within the bounds of the cart. Each cart is assumed to be of a special material, elastic enough to adapt to the current bounds of the associated nested graph. Thus, as nodes of a nested graph are pushed outwards, expanding the nested graph, the parent node adjusts its bounds accordingly. Similarly, should the bounds of the nested graph shrink, so will the geometry of the parent node by the same amount.

- Each nested graph, including the root graph, is assumed to have a dynamic (with respect to its graph bounds) center of gravity, pulling all its nodes in, towards its center, so as to keep them together, disallowing arbitrary drifts from the center. The strength of this force is independent from the size of the node and the distance between node center and graph center. Gravitational forces are assumed to be relatively weaker than spring and repulsion forces.

- For simplicity and improved efficiency, two nodes repel each other only if they are within the same graph.

- Inter-graph edges are treated specially; the part of an inter-graph edge $e$, if any, from its end-node $u$ in a nested graph $G_{u}$ to the boundary of $G_{u}$ is represented by a constant force (similar to gravitational forces), instead of a spring, so as to keep $u$ as close to the boundary of $G_{u}$ as possible. The remaining part of the inter-graph edge is represented with a regular spring. Such special treatment requires heavy computation. As the nesting tree gets deeper, the average number of graphs spanned by an inter-graph edge increases; the computational cost required to accurately implement this model will raise dramatically. However, it is possible to approximate this model by increasing the desired length of an inter-graph edge with an amount proportional to the sum of the depths of its end-nodes from their common ancestors in the nesting tree. The latter strategy has been shown to be as effective as the original schema in terms of quality and has yielded a much better running-time performance.

Fig. 4. Part of a sample compound graph (left) and the corresponding physical model used by our algorithm, where the deeper a node is in the nesting hierarchy the lighter it is colored (right). 
Fig. 4 illustrates the basics of our physical model with an example.

Notice that our approach does not impose any particular force model or set of formulas. Similarly an implementation is not confined to a specific convergence schema. Our implementation, however, was mostly based on that of Fruchterman and Reingold [13].

\subsection{Application-specific constraints}

Today's sophisticated graph visualization applications require specific constraints to be integrated into layout algorithms. These constraints may vary arbitrarily, however common examples include keeping the relative position of a group of nodes fixed and clustering a set of nodes that share a common property worth displaying [2]. However, such constraints generally introduce conflicting goals, even with the core target of the basic spring embedder itself (minimal node-node overlap and revealing symmetries).

We propose introducing additional forces to "blend" application-specific constraints into our method of drawing undirected compound graphs. In order to preserve the nice properties of the core spring embedder, in case of conflict, the default forces should govern such additional forces. Thus, application-specific forces are set to have constants of relatively smaller factors.

As a case study, let us consider the Patika editor. PATIKA [6], a pathway database and tool, is composed of a server-side, scalable, database and client-side editors to provide an integrated, multi-user environment for visualizing and manipulating a network of cellular events. PATIKA is mainly intended for signaling pathways whose underlying graph structure can be arbitrarily more complicated and irregular than that of metabolic pathways.

For a biological pathway drawing, it is quite important to group the products, substrates, and effectors of a reaction. Hence, we apply relativity constraint forces or simply relativity forces on each substrate, product, and effector states to position them properly around their associated transition(s). The convention is to align the substrates and products of a transition on opposite sides of the transition to form a certain flow direction. Effector edges, on the other hand, are left free. When calculating relativity forces, we first determine a flow, called orientation, for each transition by simply looking at the current, relative positions of their associated substrates and products. Then, each associated state of the transition is applied a relativity force to respect this orientation (Fig. 5).

Another application-specific constraint of PATIKA is due to the cellular locations of biological nodes, called compartments. A layout algorithm must keep each biological node within the bounds of the associated compartment and must enlarge or shrink it as required by the geometry of the enclosed part of the pathway.

The algorithm represents each compartment with a rectangular region and treats them similarly to an expanded node; however, unlike an expanded node, a compartment neighbors one or more other compartments, and a change in its geometry affects its neighbors. Thus, a special mechanism to resize a compartment needs to be performed. Fig. 6 shows the layout of compartments within a cell assumed by our algorithm and used by the PATIKA editor.

Finally, bond edges that represent the binding relations between members of a molecular complex are conventionally shorter than other interaction edges; hence, we set their spring constants to be smaller.

Fig. 18 shows a sample biological pathway drawing produced by the layout algorithm, as implemented within the PATIKA editor.

\subsection{Algorithm}

We assume that the graph to be laid out is represented with a graph manager object $M=(S, I, F)$, where each graph $G=(V, E)$ in $S$ is implemented using an adjacency list representation, and $V^{M}$ and $E^{M}$, respectively, represent all nodes (both simple and compound) and edges (both intra and inter-graph) in graph manager $M$. These objects can be referenced through

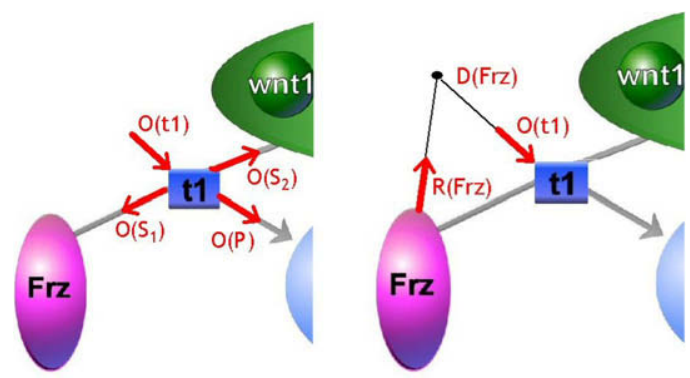

Fig. 5. An example of how the orientation of a transition is determined, shown on transition t1 of Fig. 1 (left) and how it is used to calculate the relativity force on one of its substrates, Frz (right). $\mathrm{O}(\mathrm{t} 1), \mathrm{R}(\mathrm{Frz})$, and $\mathrm{D}(\mathrm{Frz})$, respectively, denote the orientation of $\mathrm{t} 1$, relativity force on Frz due to $\mathrm{t} 1$, and desired location of Frz to obey this force, where the magnitude of $\mathrm{R}(\mathrm{Frz})$ is equal to that of $\mathrm{O}(\mathrm{t} 1)$, and the distance of $\mathrm{D}(\mathrm{Frz})$ from $\mathrm{t} 1$ is equal to the desired edge length. 


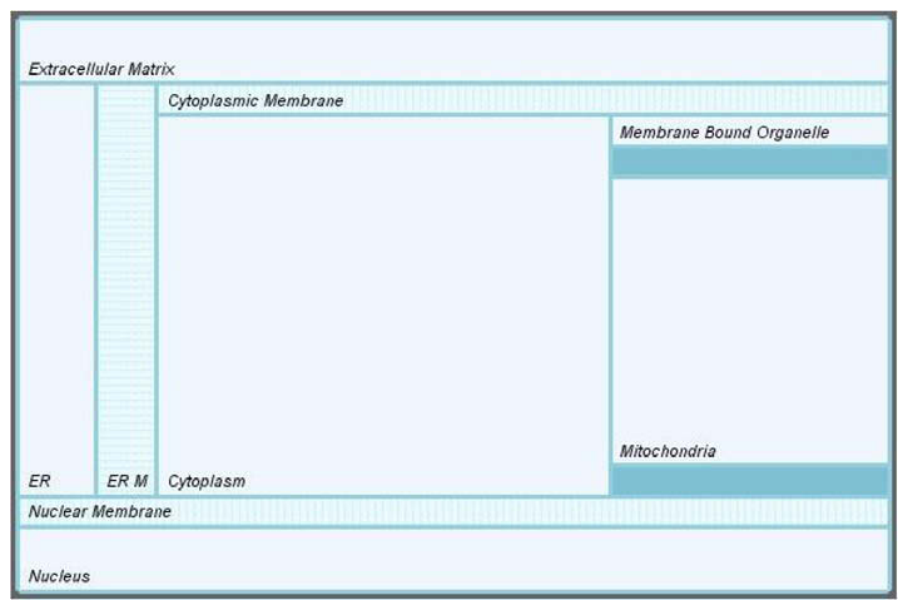

Fig. 6. The cell model assumed by our algorithm and used by PAтIKA. Each biological node is confined to its compartment.

structures named GraphMgr, Graph, Node, and Edge. Layout specific data and functionality are assumed to be kept in these structures as well.

The algorithm is composed of three major phases preceded by an initialization phase:

- Initialization: This is where initial node sizes, and threshold values for determining convergence (based on number of nodes) are calculated, and the random initial positioning of nodes is performed.

In addition, for efficiency and layout quality reasons, parts of the given graph that are trees are temporarily removed. In other words, a root graph's leaf nodes are iteratively removed until no such node is left. The remaining part of the graph forms the "skeleton" of the graph (see Fig. 7).

The overall time complexity of this method is $\Theta\left(\left|V^{M}\right|\right)$, as each node is visited $\Theta(1)$ times.

- Phase 1: In this phase, the skeleton graph is laid out using the spring embedder model described earlier, but application constraints and gravitational forces are disabled.

- Phase 2: Trees reduced earlier in the initialization phase are introduced back, level by level, in this phase, also taking application constraints and gravitational forces into account.

- Phase 3: This phase is the stabilization phase, where we "polish" the layout.

The formula for calculating the spring force for edge $e=(u, v)$ is

$$
F_{s}=\frac{\left(\lambda-\left\|p_{u}-p_{v}\right\|\right)^{2}}{\eta} p_{u} \vec{p}_{v}
$$

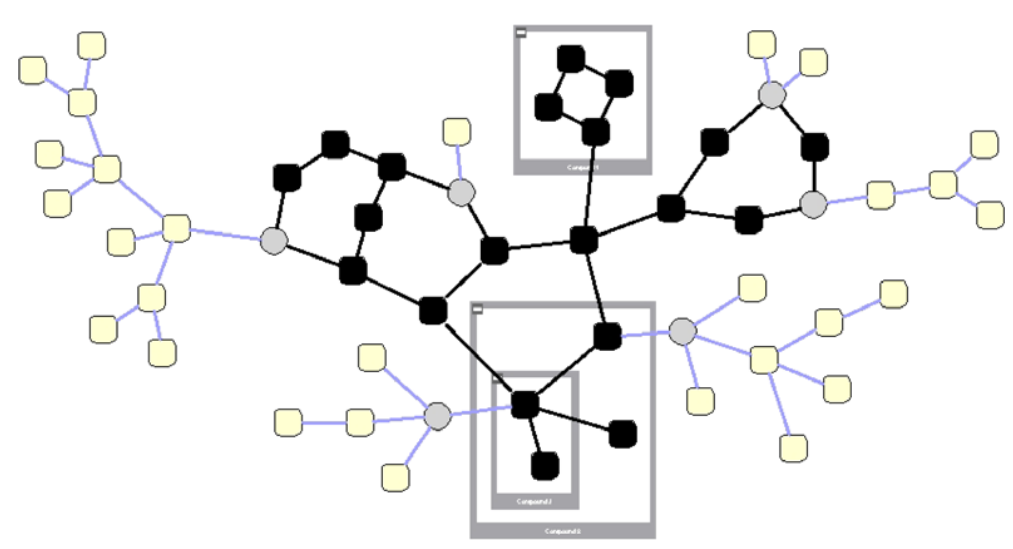

Fig. 7. The skeleton is shown dark, and reduced trees are marked with light color. Notice that only the trees that are members of the root graph are allowed to be reduced. Reduced tree roots are shown with circle nodes, as they will be the tree growth origins for later phases of the algorithm, where trees are grown in level-order. 
where $\lambda$ is the ideal edge length, $\eta$ is the elasticity constant of the edge, and $p_{u}$ and $p_{v}$ are positions of nodes $u$ and $v$, respectively. The ideal edge length of an inter-graph edge is increased proportional to the sum of the depths of its end-nodes from their common ancestors in the nesting tree. Non-uniform node dimensions require force calculations to be based on clipping points rather than node centers. The following method is used for calculating the spring forces acting on each edge's ends:

Algorithm. CalculateSpringForces(GraphMgr M)

(1) for $e=(u, v) \in E^{M}$ do

(2) idealLength $:=\lambda$

(3) if $e$ is an inter-graph edge then

(4) idealLength $*=($ u.depth $+v$.depth $) *$ NESTING_FACTOR

(5) $c_{u}:=\operatorname{LinESEGMENT}($ u.center, v.center $) \cap$ u.boundRect

(6) $\quad c_{v}:=\operatorname{LINESEGMENT}($ u.center, $v$.center $) \cap v$.boundRect

(7) $\quad F_{s}:=\left(\text { idealLength }-\left\|c_{u}-c_{v}\right\|\right)^{2} / \eta \cdot \vec{c}_{u} \vec{c}_{v}$

(8) $F_{s}(u)+=F_{s}$

(9) $\quad F_{s}(v)-=F_{s}$

The overall time complexity of this method is $\Theta\left(\left|E^{M}\right|\right)$, as all steps inside the for-loop can be processed in $\Theta(1)$ steps. Node-to-node repulsion forces are calculated using the formula

$$
F_{r}=\frac{\alpha}{\left\|p_{u}-p_{v}\right\|^{2}} p_{u} \vec{p}_{v}
$$

where $\alpha$ is the repulsion constant. Similar to spring forces, repulsion forces require us to make clipping point calculations for nodes of non-uniform size, based on the lines passing through nodes' centers:

Algorithm. ApplyRepulsionForces(GraphMgr M)

(1) $S:=\{\}$

(2) for $u \in V^{M}$ do

(3) $S:=S \cup\{u\}$

(4) for $v \in V^{M}-S$ do

(5) $\quad c_{u}:=\operatorname{LINESEGMENT}($ u.center, $v$.center $) \cap u$.boundRect

(6) $\quad c_{v}:=\operatorname{LINESEGMENT}($ u.center, $v$.center $) \cap v$.boundRect

(7) if $u \& v$ in same graph and $\left\|c_{u}-c_{v}\right\|<$ REPULSION_RANGE then

(8) $\quad F_{r}:=\alpha /\left\|c_{u}-c_{v}\right\|^{2}$

(9) $F_{r}(u)+=F_{r}$

$$
F_{r}(v)-=F_{r}
$$

Steps 5-10 are handled in $\Theta(1)$ steps, which are executed a total of a maximum $O\left(\left|V^{M}\right|^{2}\right)$ times, making the overall complexity of the method $O\left(\left|V^{M}\right|^{2}\right)$. However, because two nodes affect each other only when they are below a certain geometric distance and within the same graph, the average complexity is expected to be asymptotically lower than this.

Gravitation forces have a fixed magnitude, and they are always towards the center of the bounding rectangle of the owner graph:

Algorithm. ApplyGravitationForces(GraphMgr $M$ )

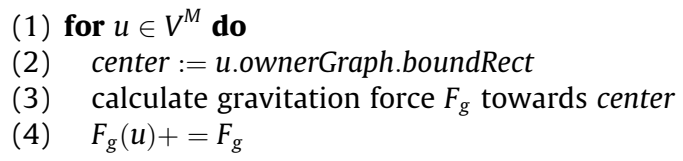

The overall time complexity of this method is $\Theta\left(\left|V^{M}\right|\right)$, as all steps inside the for-loop can be processed in $\Theta(1)$ time. Once all forces have been calculated (here $F_{a s}$ represents the total of any application-specific forces) during an iteration, we move each node with respect to the total force acting upon it, accounting for a factor $\beta$ of the current temperature maintained as part of the global cooling schema. However, we limit the movement of each node in each iteration to avoid drastic movements, that often result in "oscillations". Notice that here we also assume that in each iteration, nodes are processed in a bottom-up manner in the nesting tree, where compound nodes are processed after the nodes of their child graphs: 
Algorithm. CalcNodePositionsAndSizes(GraphMgr M)

(1) for $u \in V^{M}$ in a bottom-up manner do

(2) $F_{\text {tot }}(u)=F_{s}(u)+F_{r}(u)+F_{g}(u)+F_{a s}(u) \cdot$ step $\cdot \beta$

(3) if $\left\|F_{\text {tot }}(u)\right\|>$ MAX_DISPLACEMENT then

(4) reduce magnitude of $F_{\text {tot }}(u)$ to MAX_DISPLACEMENT

(5) u.center $+=F_{\text {tot }}(u)$

(6) $F_{s}(u):=F_{r}(u):=F_{g}(u):=F_{a s}(u):=0$

(7) if $u$ is COMPOUND then

(8) PropogateToChildren $\left(u, F_{\text {tot }}(u)\right)$

Algorithm. PropogateToChildren(Nodeu, VectorF $\left._{\text {prop }}\right)$

(1) for each node $v$ of child graph of $u$ do

(2) $v$.center $+=F_{\text {prop }}$

(3) if $v$ is COMPOUND then

(4) $\operatorname{PropogateToChildren}\left(v, F_{\text {prop }}\right)$

(5) update bounds of child graph of $u$

The main method making use of earlier ones to implement the layout algorithm is as follows:

Algorithm. CompoundLayout(GraphMgrM $=(S, I, F))$

(1) call Initialize $(M)$

(2) phase $:=1$

(3) if layout type is incremental then//respect current positions

(4) phase $:=3$

(5) while phase $\leqslant 3$ do

(6) step $:=$ maxIterCount [phase]//use predefined iteration limits per phase

(7) error $:=0$

(8) while (step $>0$ and error $>$ errorThreshold [phase]) or !allTreesGrown do

(9) call ApplySpringForces $(M)$

(10) call Applysc epulsionForces $(M)$

(11) if phase $\neq 1$ then

(12) call ApplyGravitationForces $(M)$

(13) call ApplyAppSpecificForces $(M)$

(14) call CalcNodePositionsAndSizes $(M)$

(15) if phase $=2$ and ! allTreesGrown and step $\%$ treeGrowingStep $=0$ then

(16) call GrowTreesOneLevel $(M) / /$ grow in BFS manner

(17) step $:=$ step -1

(18) phase $:=$ phase +1

A quick analysis of the algorithm reveals that the running-time of the layout of a compound graph is $O\left(k \cdot\left|V^{M}\right|^{2}\right)$, where $k$ is the number of iterations required to reach an energy minimal state.

\section{Implementation}

The algorithm described above has been tested within the example application of Tom Sawyer Visualization for Java, version 7.0. The development environment was Sun's Java SDK 1.4 and Microsoft Windows XP operating system on an ordinary personal computer (Pentium IV with $2 \mathrm{GHz}$ CPU and $512 \mathrm{MB}$ memory). The results were found quite satisfactory, as far as the general graph drawing criteria, such as number of crossings, and total area are concerned (Figs. 8 and 9). Furthermore, the experimental executions were found to be not only reasonably fast for interactive use but also in line with the earlier theoretical analysis, as detailed below.

\subsection{Experimental results}

We performed experiments on the execution time of our layout algorithm on randomly generated graphs with one of several parameters changing for each set. For each test, a random graph manager to be laid out was generated with the provided parameters: 

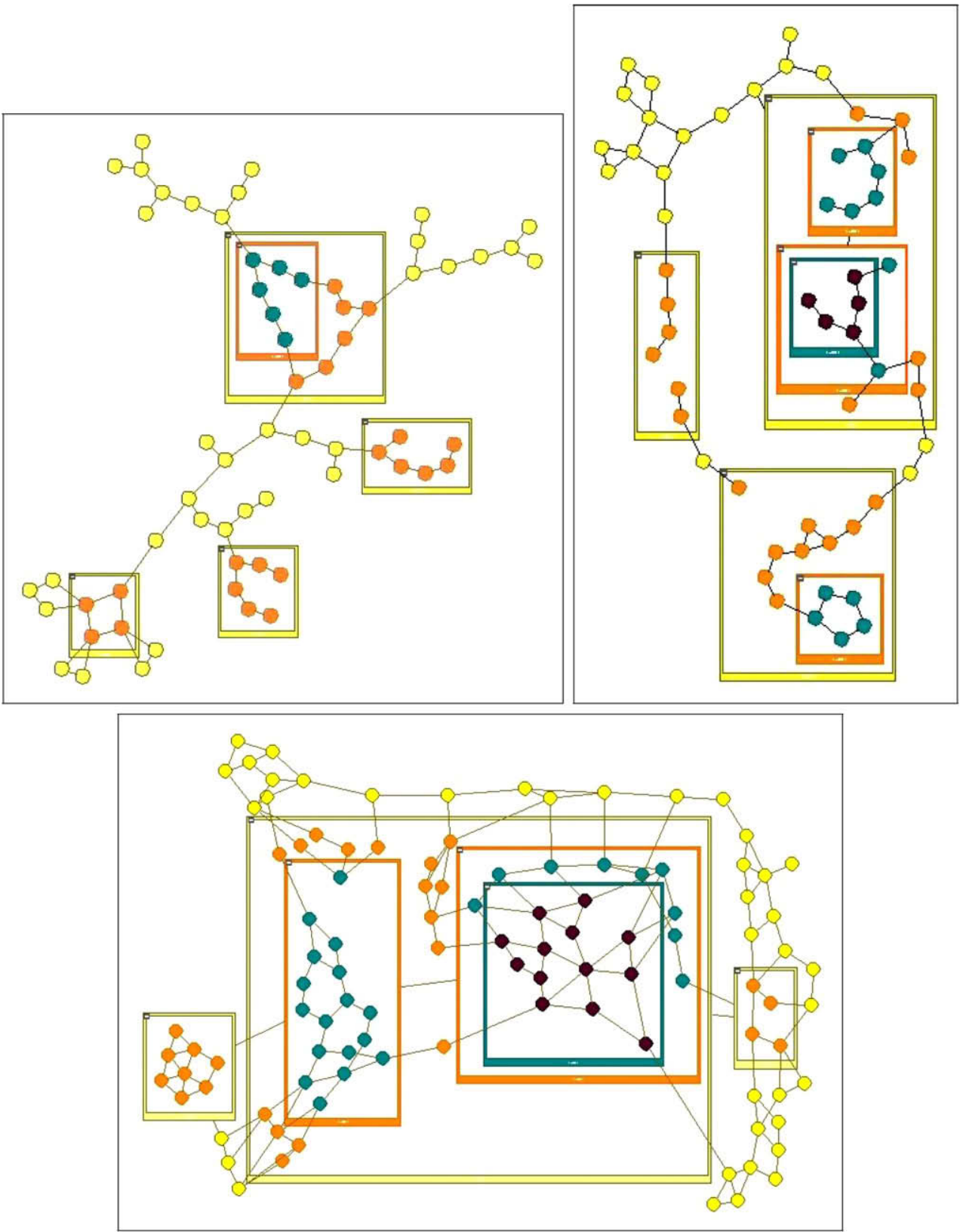

Fig. 8. Sample compound graphs (with varying desired edge lengths and edge and inter-graph edge density) laid out by our algorithm. The nodes are colorcoded to denote the depth of the node in the nesting hierarchy (i.e., the deeper a node is, the darker its color is).

- $n$ : total number of nodes,

- $m / n$ : proportion of number of edges to nodes; the number of edges is assumed to be linear in the number of nodes,

- $m_{i g} / m$ : proportion of inter-graph edges to number of all edges,

- $d$ : maximum nesting depth,

- $b$ : maximum branching (i.e., number of children of a node) in the nesting tree,

- $p$ : probability of pruning a child in the nesting tree to avoid nesting trees that are too uniform in structure.

First we construct a nesting tree and a graph manager that realizes this nesting structure with the specified parameters. Then, the nodes are created and distributed to graphs in the graph manager uniformly. Similarly, end-nodes of each edge are picked randomly. Each test is executed 10 times, and the average is taken. For simplicity, we take the dimensions of leaf nodes to be uniform, even though our algorithm is able to handle non-uniform dimensions for not only non-leaf (compound) nodes but also leaf nodes. Fig. 10 shows an example of a randomly generated graph. 


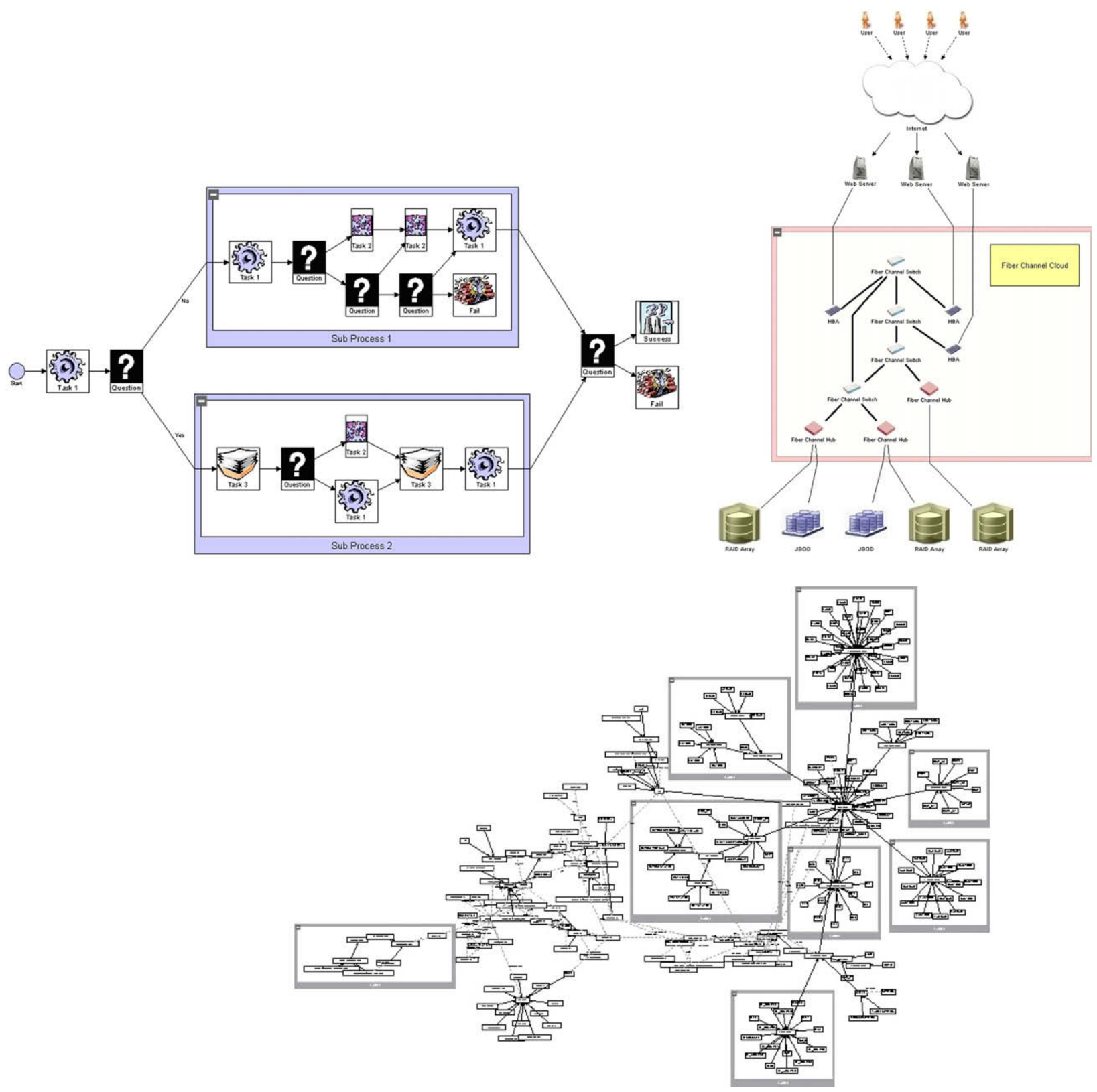

Fig. 9. Sample real-life compound graphs (with varying desired edge lengths and node sizes) from business workflow, networking, and software modeling (courtesy of Tom Sawyer Software), laid out with our algorithm.

From the theoretical analysis given earlier, a quadratic behavior of execution time is expected, assuming $k$ does not grow in the order of the graph size. The experiments validate this argument (Fig. 11).

We also experimented with the nesting depth (Fig. 12). The experiments show that initially deeper nesting helps improve execution time, as the number of nodes per graph decreases, due to the fact that certain calculations such as node-to-node repulsion forces are only performed within each graph. However, as the nesting depth increases, the performance decreases dramatically, due to the increase in the number of compound nodes and nested graphs.

Furthermore, we performed a test set to see how the proportion of inter-graph edges to regular edges affects the execution time (Fig. 13). As expected, the time it takes to process an inter-graph edge as opposed to a regular edge does not vary much.

In addition, we wanted to see how the average number of nested graphs per graph affected the execution time (Fig. 14). Again, initially deeper nesting helps decrease the execution time, because some expensive calculations are then performed in a divide-and-conquer fashion. However, as the nesting becomes even deeper, the time it takes to process more compound nodes and deeper nodes dominates, and the execution gets slower. 


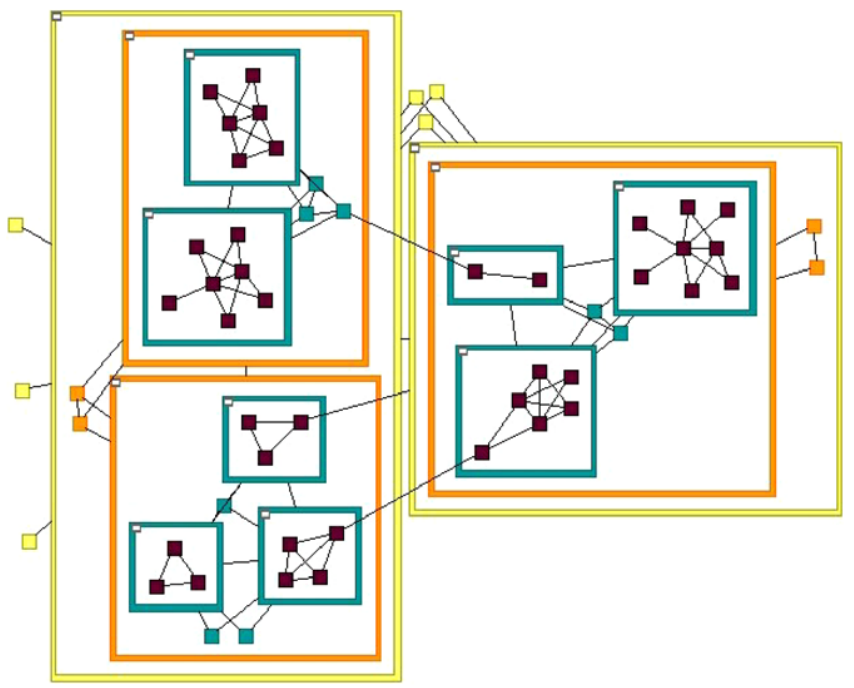

Fig. 10. A randomly generated graph laid out by our algorithm. $\left(n=70, m / n=1.5, m_{i g} / m=0.03, d=3, b=3\right.$, and $\left.p=0.33\right)$.

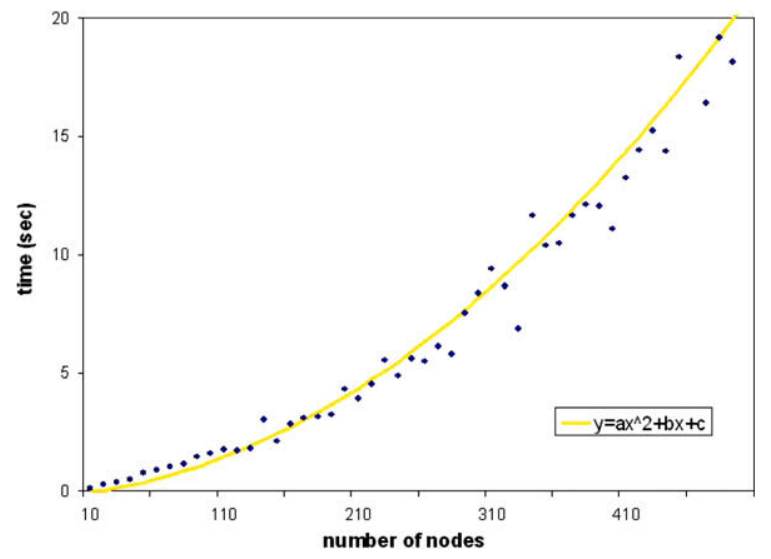

Fig. 11. Number of nodes $(n)$ vs. execution time of our algorithm. $\left(m / n=1.5, m_{i g} / m=0.05, d=3, b=3\right.$, and $\left.p=0.33\right)$.

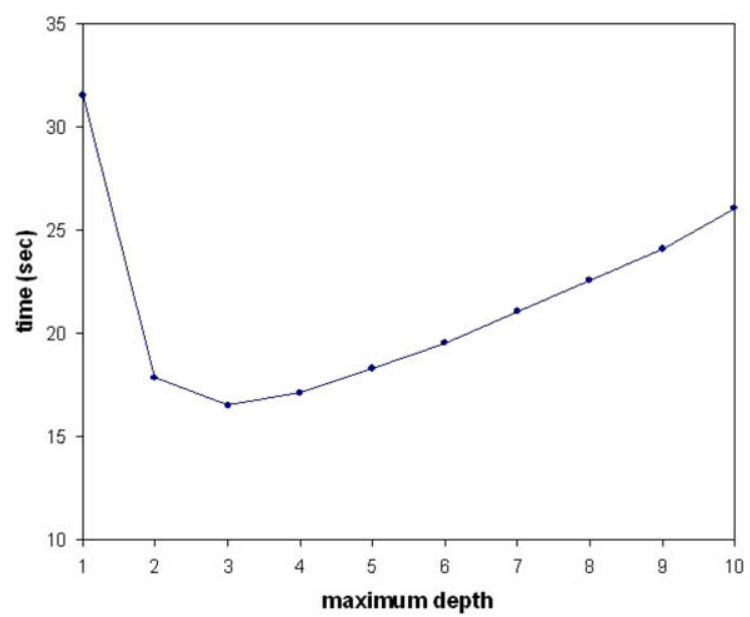

Fig. 12. Maximum nesting depth $(d)$ vs. execution time of our algorithm. $\left(n=500, m / n=1.5, m_{\text {ig }} / m=0.05, b=3\right.$, and $\left.p=0.33\right)$. 


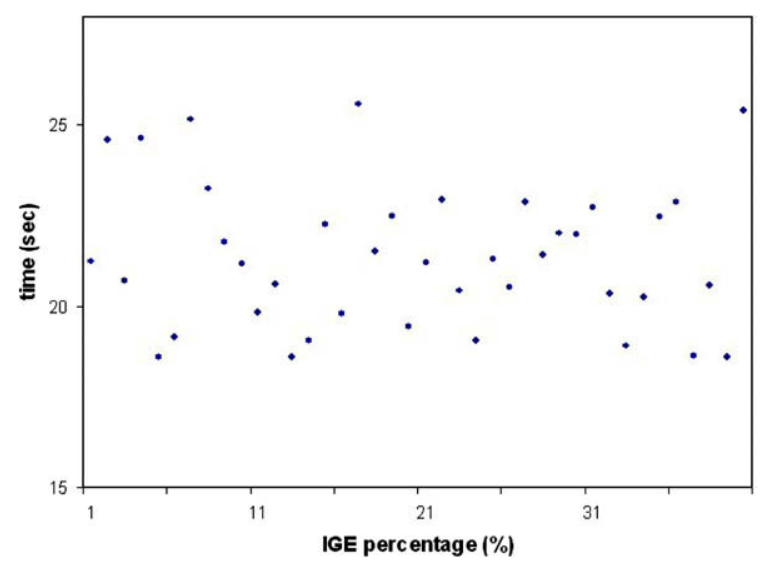

Fig. 13. Proportion of inter-graph edges to all edges $\left(m_{i g} / m\right)$ vs. execution time of our algorithm. $(n=500, m / n=1.5, d=3, b=3$, and $p=0.33)$.

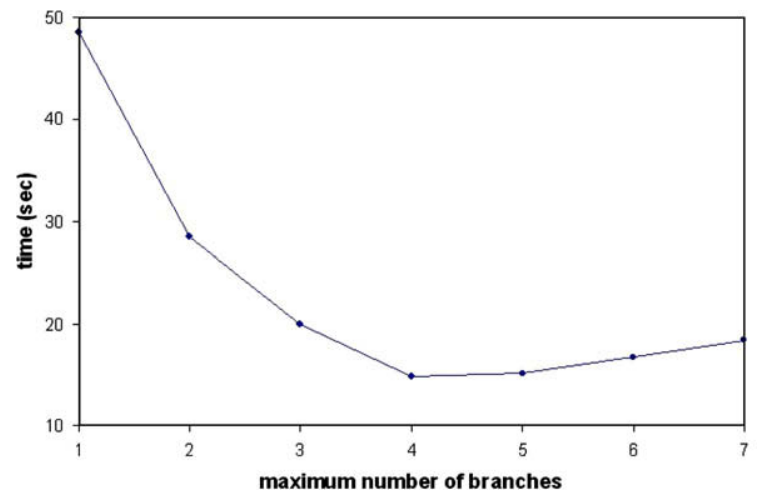

Fig. 14. Maximum branching in the nesting tree $(b)$ vs. execution time of our algorithm. $\left(n=500, m / n=1.5, m_{i g} / m=0.05, d=3\right.$, and $p=0.33$ ).
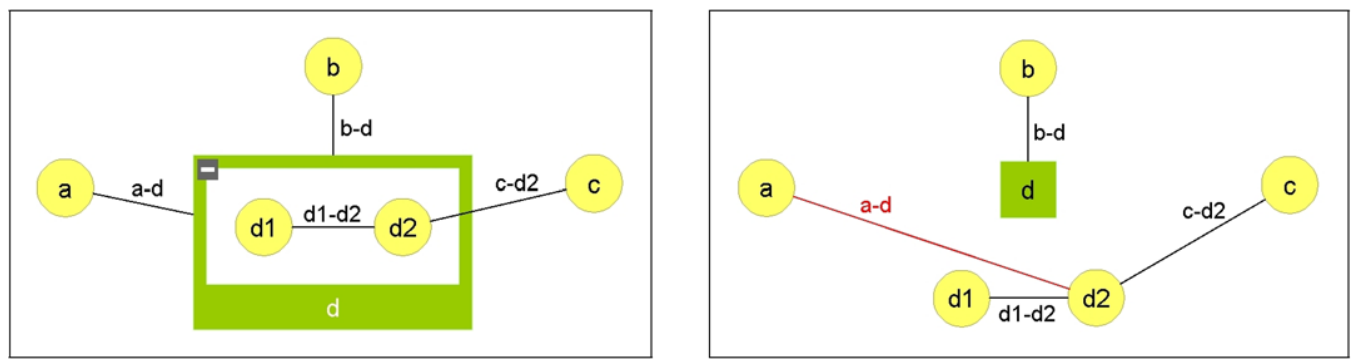

Fig. 15. An example of converting a compound graph into a non-compound one by recursively taking nested contents of a compound node outside while reconnecting some edges (edge "a-d" in this case) of the compound node to the previously nested nodes.

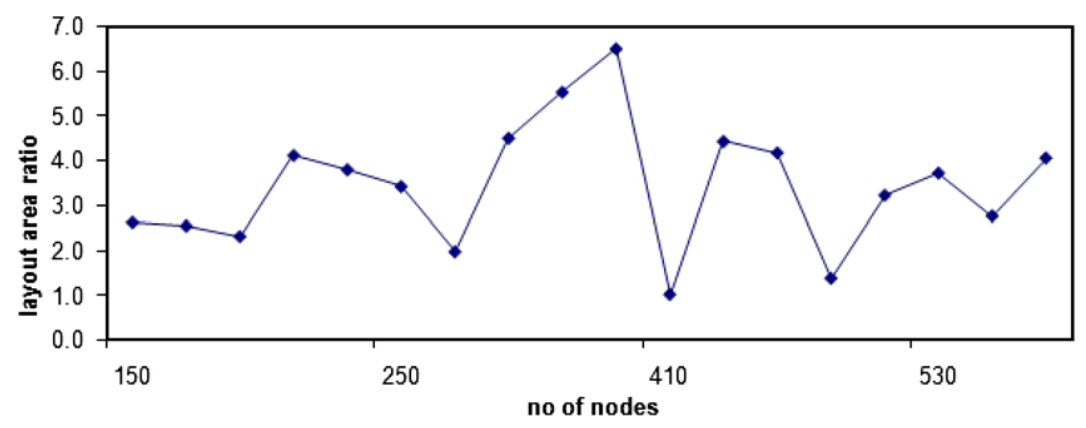

Fig. 16. Ratio of the layout area for randomly created compound and non-compound graphs. 


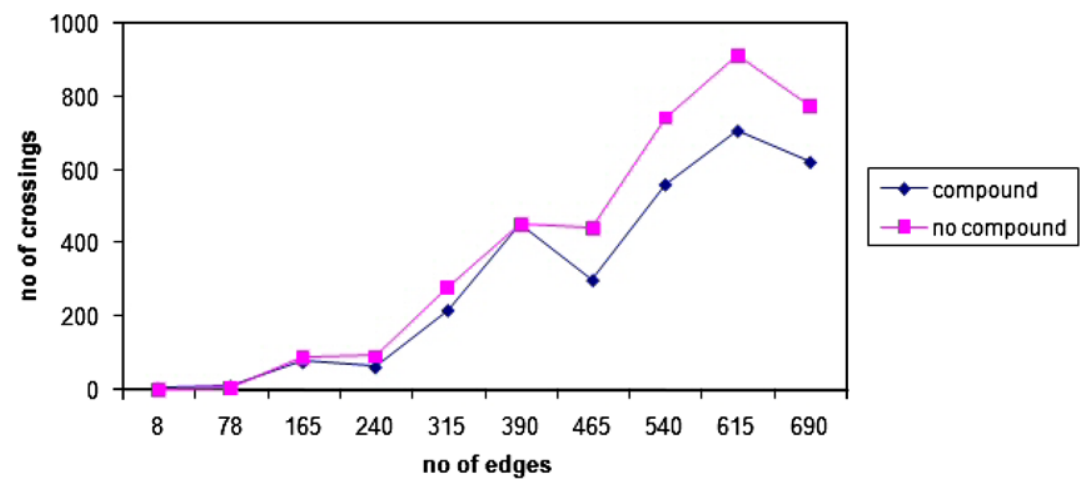

Fig. 17. Comparison of the number of edge crossings for compound and associated non-compound graphs created randomly.

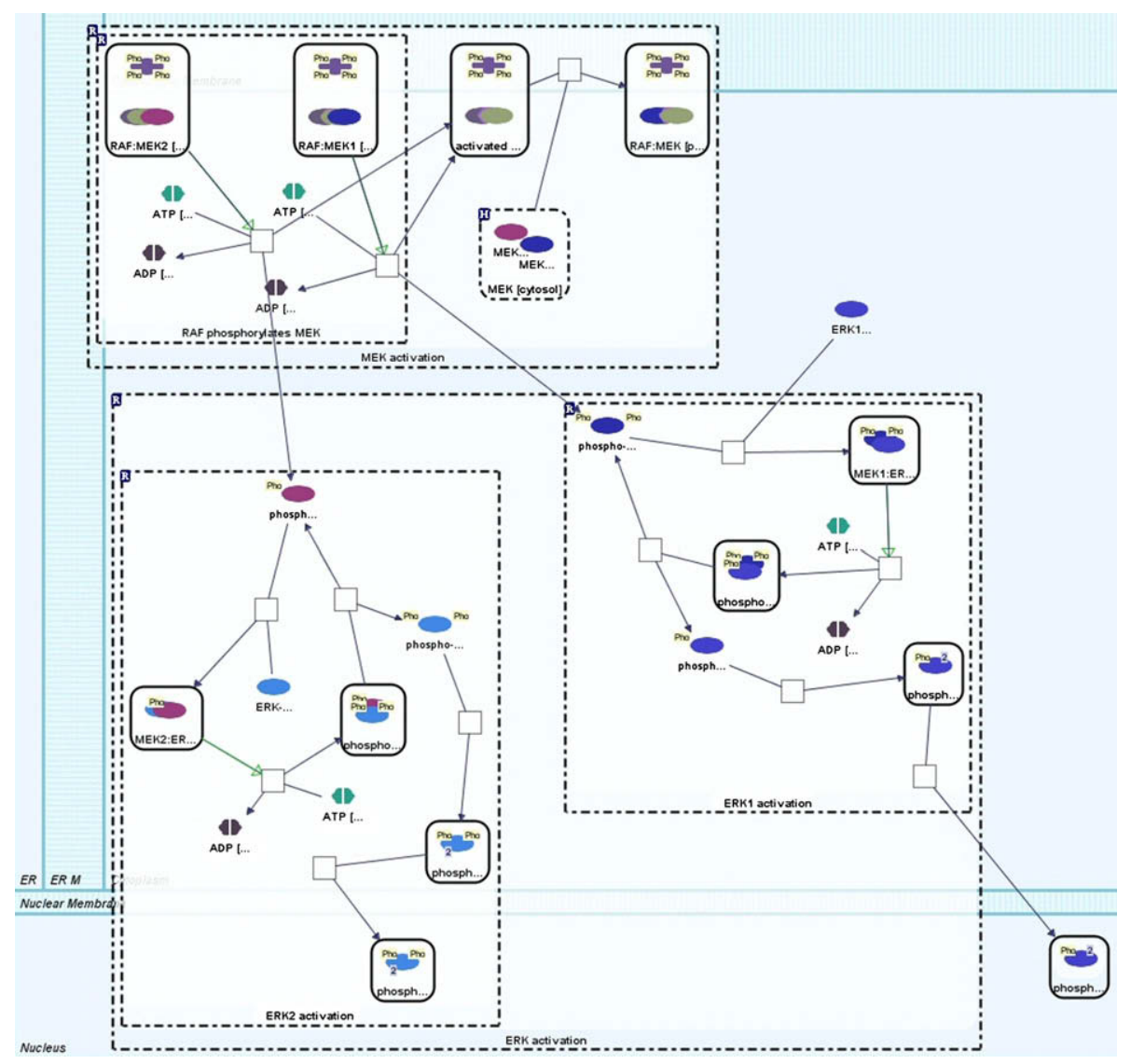

Fig. 18. A sample pathway (obtained by a query from the PATIKA database) laid out by our algorithm.

We also performed certain tests for measuring the quality of the resulting drawings.

The first set of quality tests were performed to check for node-to-node overlaps. It is extremely hard to completely eliminate node overlaps for drawings of non-uniform node dimensions that are generated by a spring embedder, due to the fact that the constants associated with opposing attraction and repulsion forces are difficult to fine-tune. The results yielded nodeto-node overlaps of only, at most, one node pair in a thousand, and the overlap amounts are almost always inconsequential.

Other quality metrics we employed for measurement include area and edge crossings. For this purpose, we compared the quality metrics of random compound graphs with non-compound graphs that were constructed from the compound ones, trying to keep the topology as similar to the original as possible, as follows: recursively transfer out to the root graph the contents of the child graph of each compound node in the root graph, which converts the compound node into a simple node, until no compound nodes are left. During this process, also reconnect some (roughly half) incident edges of the compound 


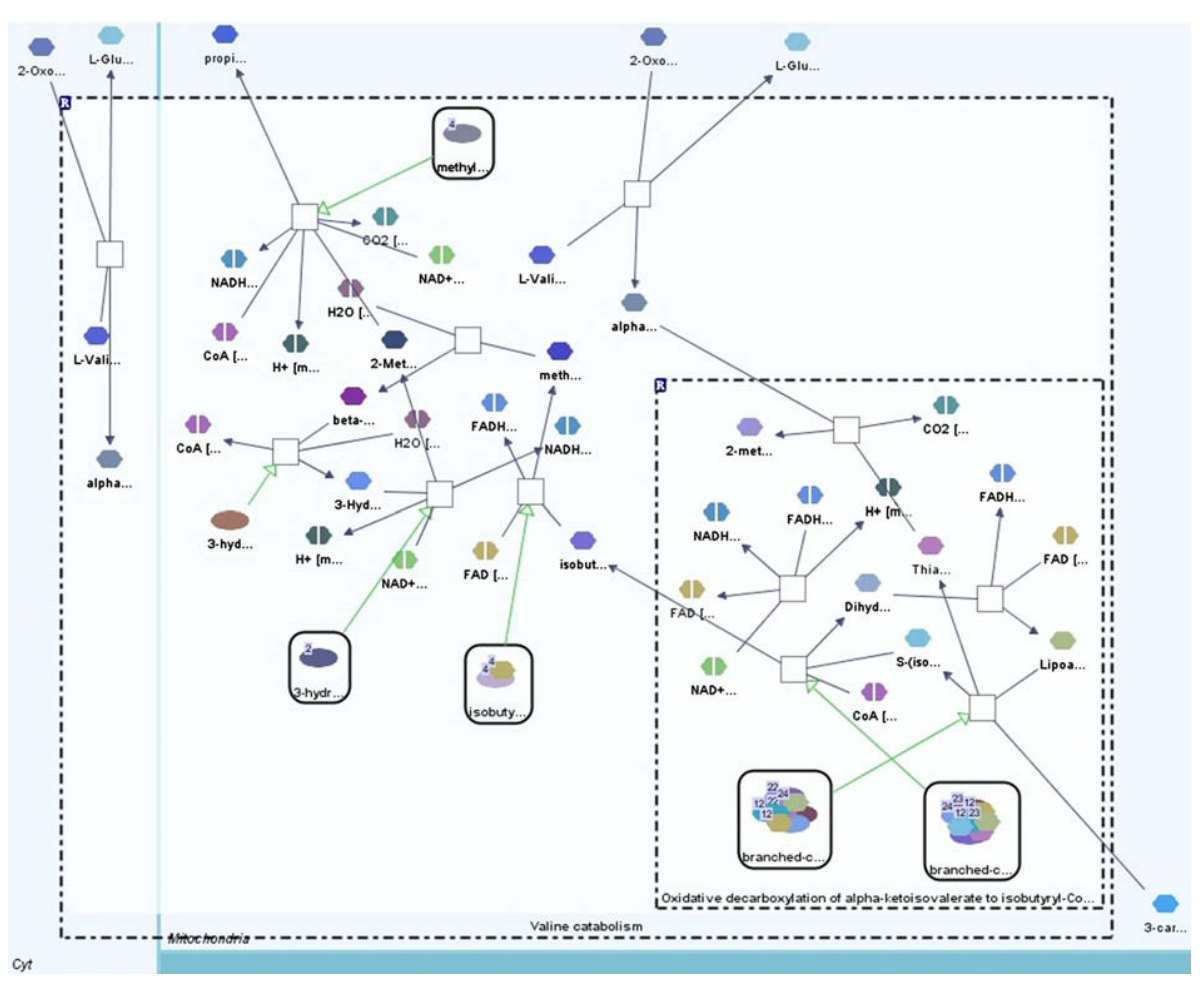

Fig. 19. A sample pathway laid out by our algorithm.

node to the nodes inside its child graph. Fig. 15 shows an example conversion. Random graphs used for this purpose were sized from 10 to 750 nodes.

We measured and compared the area occupied by the resulting drawings for randomly created compound and non-compound graphs, as explained earlier. It turns out that the ratio of the area occupied by a compound graph after layout is roughly a few folds of the area occupied by the drawing obtained from the associated non-compound graph (Fig. 16). This was expected due to the extra space introduced by compound nodes and their margins.

Another test that was performed for measuring the quality was for the number of crossings. The number of crossings for tested compound graphs has always been less than the associated non-compound graph, within a small constant factor, signifying that final positions of the nodes at the end of our compound graph layout algorithm yields the structure of the underlying topology as well as a regular spring embedder based layout (Fig. 17).

\subsection{An application}

We also implemented our algorithm as part of a new version of the PAтIKA pathway editor. In this implementation, method APPLyAPPSPECIFICForces mentioned earlier was defined as follows, to satisfy the relative placement constraints of the substrates and products of a particular reaction:

Algorithm. AptlyApPSecificForces $($ Edgee $=(u, v))$

(1) if phase $\geqslant 2$ then

(2) orientation $:=$ e.transition.orientation

(3) if e is substrate then

(4) orientation := -orientation

(5) Calculate $F_{r c}$ on $e$ according to its orientation

(6) $\quad F_{r c}(u)+=F_{r c}$

(7) $\quad F_{r c}(v)-=F_{r c}$

The results was found to be satisfactory, as far as the general graph drawing criteria, as far as the number of crossings and total area are concerned. In addition, application-specific constraints, such as compartmental constraints and relative positioning constraints, seem to be highly satisfied. Figs. 18 and 19 show sample pathway drawings that were produced. Notice that the subcellular location (i.e., compartments) of biological nodes are respected as well as grouping (i.e., nesting), and compartments are resized to tightly fit their contents. 


\subsection{Implementation issues}

The use of "momentum" or "temperature" for each node [11] has helped the convergence greatly. Each node's movement is not only based on the total force calculated during the current iteration but also on the previous one. For simplicity and efficiency reasons, we simply added a constant portion of the previous iteration's total force to this iteration's total force for each node, resulting in dramatic improvements in execution times.

Another quick improvement was due to the use of a range for repulsion forces; thus, repulsion forces were calculated only if the nodes were within a certain distance.

\section{Conclusion}

We presented a novel algorithm for the layout of undirected compound graphs. This is the first spring embedder that can handle compound graphs without any restriction on topology or geometry. The layout of complicated pathway graphs, such as those in PATIKA, are among the target applications. The main novelties of our work include the use of a modified spring embedder system that treats compound nodes and inter-graph edges as part of the physical system. In addition, we believe that most application-specific drawing conventions, such as those in biological pathways, can be integrated into this physical system as additional forces, as long as they can be sketched out as part of the physical model described. Experimental results are found satisfactory both in terms of the quality of layouts and computational efficiency.

\section{References}

[1] F. Bertault, M. Miller, An algorithm for drawing compound graphs, in: Graph Drawing (Proc. GD'99), Lecture Notes in Computer Science, vol. 1731, Springer-Verlag, 1999, pp. 197-204.

[2] K. Bohringer, F. Paulisch, Using constraints to achieve stability in automatic graph layout algorithms, in: CHI'90 Proceedings, ACM, 1990 , pp. $43-51$.

[3] G. Di Battista, W. Didimo, A. Marcandalli, Planarization of clustered graphs, in: Graph Drawing (Proc. GD’01), Lecture Notes in Computer Science, vol. 2265, Springer-Verlag, 2001, pp. 60-74.

[4] G. Di Battista, P. Eades, R. Tamassia, I.G. Tollis, Graph Drawing, Algorithms for the Visualization of Graphs, Prentice-Hall, 1999.

[5] E. Di Giacomo, W. Didimo, L. Grilli, G. Liotta, Graph visualization techniques for web clustering engines, IEEE Transactions on Visualization and Computer Graphics 13 (2) (2007) 294-304.

[6] U. Dogrusoz, E. Erson, E. Giral, E. Demir, O. Babur, A. Cetintas, R. Colak, PATIKAweb: a Web interface for analyzing biological pathways through advanced querying and visualization, Bioinformatics 22 (3) (2006) 374-375.

[7] U. Dogrusoz, Q. Feng, B. Madden, M. Doorley, A. Frick, Graph visualization toolkits, IEEE Computer Graphics and Applications 22 (1) (2002) 30-37.

[8] P. Eades, A heuristic for graph drawing, Congressus Numerantium 42 (1984) 149-160.

[9] P. Eades, Q. Feng, X. Lin, Straight-line drawing algorithms for hierarchical graphs and clustered graphs, in: S. North (Ed.), GD'96, Lecture Notes in Computer Science, vol. 1190, Springer-Verlag, 1997, pp. 113-128.

[10] P. Eades, M. Huang, Navigating clustered graphs using force-directed methods, Journal of Graph Algorithms and Applications 4 (3) (2000) 157-181.

[11] A. Frick, A. Ludwig, H. Mehldau, A fast adaptive layout algorithm for undirected graphs, in: R. Tamassia, I. Tollis (Eds.), GD'94, Lecture Notes in Computer Science, vol. 894, Springer-Verlag, 1995, pp. 388-403.

[12] Y. Frishman, A. Tal, Dynamic drawing of clustered graphs, in: Proceedings of IEEE Symposium on Information Visualization, 2004, pp. 191-198.

[13] T.M.J. Fruchterman, E.M. Reingold, Graph drawing by force-directed placement, Software Practice and Experience 21 (11) (1991) $1129-1164$.

[14] K. Fukuda, T. Takagi, Knowledge representation of signal transduction pathways, Bioinformatics 17 (9) (2001) $829-837$.

[15] D. Harel, Y. Koren, Drawing graphs with non-uniform vertices, in: Working Conference on Advanced Visual Interfaces (Proc. AVI'02), ACM Press, 2002, pp. 157-166.

[16] W. Lai, P. Eades, A graph model which supports flexible layout functions, Tech. Rep. 96-15, Callaghan 2308, Australia, 1996.

[17] M. Raitner, HGV: A library for hierarchies, graphs, and views, in: M. Goodrich, S. Kobourov (Eds.), Proc. Graph Drawing'02, LNCS, vol. 1528, 2002, pp. 236-243.

[18] G. Sander, Layout of compound directed graphs, Tech. Rep. A/03/96, University of Saarlandes, CS Dept., Saarbriicken, Germany, 1996.

[19] K. Sugiyama, K. Misue, Visualization of structural information: automatic drawing of compound digraphs, IEEE Transactions on Systems, Man and Cybernetics 21 (4) (1991) 876-892.

[20] K. Sugiyama, K. Misue, A generic compound graph visualizer/manipulator: D-ABDUCTOR, in: F.J. Brandenburg (Ed.), GD'95, Lecture Notes in Computer Science, vol. 1027, Springer-Verlag, 1995, pp. 500-503.

[21] X. Wang, I. Miyamoto, Generating customized layouts, in: F. Brandenburg (Ed.), Graph Drawing (Proc. GD’95), Lecture Notes in Computer Science, vol. 1027, Springer-Verlag, 1995, pp. 504-515. 